International Journal of Pure and Applied Mathematics

Volume 103 No. 2 2015, 311-320

ISSN: 1311-8080 (printed version); ISSN: 1314-3395 (on-line version)

url: http://www.ijpam.eu

doi: http://dx.doi.org/10.12732/ijpam.v103i2.15

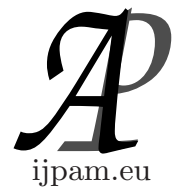

\title{
CHAOS CONTROL VIA STATE-DEPENDENT RICCATI EQUATION METHOD APPLIED A NON-IDEAL VIBRATION ABSORBER COUPLED TO A NONLINEAR OSCILLATOR
}

\author{
Fábio Roberto Chavarette ${ }^{1 \S}$, Nelson José Peruzzi ${ }^{2}$, \\ Douglas da Costa Ferreira ${ }^{3}$, Mara Lucia Martins Lopes ${ }^{4}$ \\ ${ }^{1,4}$ Department of Mathematics \\ Faculty of Engineering of Ilha Solteira \\ UNESP - Univ.Estadual Paulista \\ Brasil Avenue, 56, 15385-000, Ilha Solteira, SP, BRAZIL \\ ${ }^{2}$ Department of Exact Sciences \\ UNESP - Univ. Estadual Paulista \\ Via de Acesso Prof. Paulo Donato Castellane s/n, \\ 14884-900 - Jaboticabal, SP, BRAZIL \\ ${ }^{3}$ Mechanical Engineering Department \\ UFMT - Federal University of Mato Grosso \\ Rondonópolis, MT, BRAZIL
}

\begin{abstract}
The non-ideal vibration absorber system coupled to a nonlinear oscillator is proposed in the paper. This system is demonstrated with a chaotic behavior and we present the control technical for the chaos stabilization. The simulation results show the effectiveness of the control strategy.
\end{abstract}

AMS Subject Classification: $93 \mathrm{C} 10$

Key Words: chaos control, state-dependent Riccati equation, non-ideal system, vibration

Received: May 13, 2015

(C) 2015 Academic Publications, Ltd. url: www.acadpubl.eu

${ }^{\S}$ Correspondence author 


\section{Introduction}

In recent decades the control of structural vibration has become increasingly more interest from engineers and researchers because of the fact that these vibrations are unwanted and may cause noise emission, premature wear and fatigue failure of components. Thus there is great interest in reducing vibrations in the system through the use of control techniques $[5,6,7]$.

The structural control is a technology for protection of structures which promotes a change in the stiffness and damping properties of the structure by adding external devices or by the action of external forces. Vibration absorbers have been applied to the control (reduction) of vibrations in structures, and the absorbers are simple devices that, when properly connected to a structure, are able to promote the reduction of its vibrations in an effective manner and consequently in many cases, reduction of noise levels, with the advantage of not requiring high costs for its implementation.

The tuned mass damper(TMD)is one popular device for minimize vibrations of mechanical structures. Frahm [1] was the first to introduce the TMD concept and considered a linear attachment composed of a mass and a spring coupled to a conservative linear oscillator(LO). Viguie and Kerschen proposed in [3] the tuning of a nonlinear vibration absorber coupled to an essentially nonlinear oscillator. The dynamic model of non-ideal power source can be defined for a beam excited by an unbalanced direct current (DC) motor was proposed by Kononenko [2]. This paper proposes the control of the vibration absorber coupled to a nonlinear oscillator model,and for its development we organize the paper this way. In Section 2, we present the mathematical model. In Section 3 , the control design control project is propose for the minimize vibration and in Section 4, the conclusions are presented.

\section{Non-ideal Model}

Figure 1 shows the non-ideal and nonlinear absorber coupled to nonlinear oscillator proposed, which consist of a nonlinear oscillator composed of a mass $m_{1}$ and a cubic stiffness $k_{n l 1}$ and the absorber with a mass $m_{2}$ and a nonlinear stiffness $k_{n l 2}$. $c_{1}$ and $c_{2}$ are the weak damping to induce energy dissipation [3].

The angular position $z$ is non-ideal excitation response, $a, b$ are motor torque constants, $r$ is the distance from unbalanced mass to rotation center of the DC motor and $d$ is related to moment of inertia of the system. All parameters are dimensionless constant positives. The term $\ddot{z}$ is due interaction 


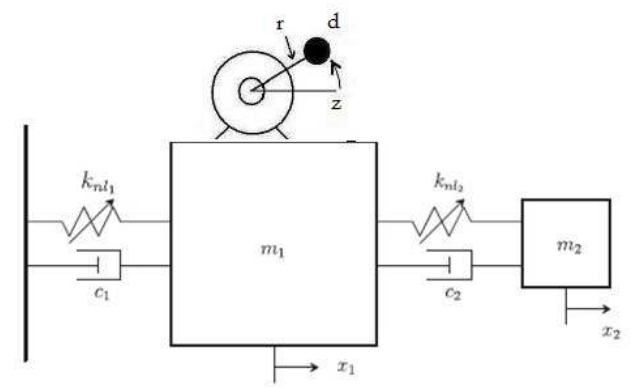

Figure 1: Non-ideal and nonlinear absorber coupled to nonlinear oscillator.

between the dynamical system and an energy source. The parameter $a$ is the applied torque constant and depends on initial conditions and $b$ is resistive net torque and has no influence of initial conditions and is considered as internal damping of the DC Motor.

The equations that describe the device illustrate in Figure 1 are:

$$
\begin{array}{r}
m_{1} \ddot{x}_{1}+c_{1} \dot{x_{1}}-c_{2}\left(\dot{x_{1}}-\dot{x_{2}}\right)+k_{n l 1} x_{1}^{3}-k_{n l 2} \\
\left(x_{1}-x_{2}\right)^{3}=d\left(z^{2} \cos \dot{z}+\ddot{z} \sin z\right), \\
m_{2} \ddot{x}_{2}+c_{2}\left(\dot{x_{2}}-\dot{x_{1}}\right) k_{n l 2}\left(x_{2}-x_{1}\right)^{3}=0, \\
\ddot{z}+b z-r \ddot{q} \sin \ddot{z}+a=0 .
\end{array}
$$

Rewriting the equations of the dynamical system, in state form, making $y_{1}=x_{1}, y_{2}=\dot{x_{1}}, y_{3}=x_{2}, y_{4}=\dot{x_{2}}, y_{5}=z$ and $y_{6}=\dot{z}$ the governing equations may be written as being:

$$
\begin{array}{r}
\dot{y}_{1}=y_{2}, \\
\dot{y}_{2}=\frac{-c_{1} y_{2}+c_{2}\left(y_{2}-y_{4}\right)-k_{n l 1} y_{1}^{3}-k_{n l 2}\left(y_{1}-y_{3}\right)^{3}}{m_{1}-d r\left(\sin ^{2} y_{5}\right)} \\
+\frac{d y_{6}^{2} \cos \left(y_{5}\right)+d \sin \left(y_{5}\right)\left(a-b y_{6}\right)}{m_{1}-d r\left(\sin ^{2} y_{5}\right)}, \\
\dot{y}_{3}=y_{4}, \\
\dot{y}_{4}=-\frac{c_{2}}{m_{2}}\left(y_{4}-y_{2}\right)-k_{n l 2}\left(y_{3}-y_{4}\right)^{3}, \\
\dot{y}_{5}=y_{6},
\end{array}
$$




$$
\begin{gathered}
\dot{y}_{6}=\frac{\left(m_{1}-d r \sin ^{2} y_{5}\right)\left(a-b y_{6}\right)+r \sin y_{5}\left(-c_{1} y_{2}+c_{2}\left(y_{2}-y_{4}\right)\right.}{m_{1}-d r\left(\sin ^{2}\left(y_{5}\right)\right)} \\
+\frac{\left(-k_{n l 1} y_{1}^{3}-k_{n l 2}(y 1-y 3)^{3}+d y_{6}^{2} \cos y_{5}+d \sin y_{5}\left(a-b y_{6}\right)\right)}{m_{1}-d r\left(\sin ^{2}\left(y_{5}\right)\right)} .
\end{gathered}
$$

Here, $x_{1}$ and $x_{2}$ are the velocity and the displacement of the tuned mass damper, $x_{3}$ and $x_{4}$ are the velocity and displacement of the nonlinear oscillator and $x_{5}$ and $x_{6}$ are the angular position of non-ideal excitation response. The Figure 2 illustrate the dynamics behavior of the adopted dynamics model, by using numerical values, for the chosen parameters $m_{1}=1 \mathrm{~kg}, m_{2}=0.05 \mathrm{~kg}, c_{1}=$ $0.002 \mathrm{Ns} / \mathrm{m}, c_{2}=0.002 \mathrm{Ns} / \mathrm{m}, k_{1}=0.5 \mathrm{~N} / \mathrm{m}$ and $k_{2}=0.025 \mathrm{~N} / \mathrm{m}$ [3] by using numerical values to the tuned mass damper coupled to a linear oscillator and $a=5 \mathrm{Nm}, b=1.5 \mathrm{Nm}, r=0.3 \mathrm{~m}$ and $d=0.2 \mathrm{kgm}^{2}$ to the non-ideal excitation [8]. The initial conditions are $x_{1}(0.1)=0, x_{2}(0)=0, x_{3}(0)=0, x_{4}(0)=0$, $x_{5}(0.5)=0$ and $x_{6}(0)=0$
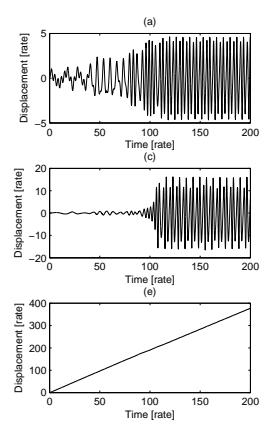

(a) Time History.
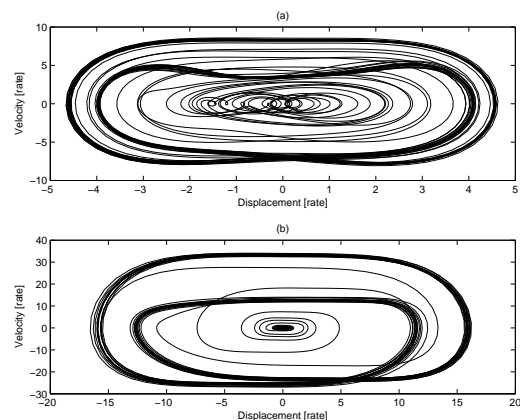

(b) Phase Portrait

Figure 2: Dynamical System

The eigenvalues of the system (2) indicates that the system is unstable, with $\lambda_{1}=-1.6016, \lambda_{2}=-0.5312, \lambda_{3}=-0.0394, \lambda_{4}=-0.0003, \lambda_{5}=0.3143+$ $0.3586 i$ and $\lambda_{6}=0.3143-0.3586 i$.

Figure 3 illustrates the Lyapunov exponents, $\lambda_{1}=0.30392 ; \lambda_{2}=0.30538$; $\lambda_{3}=-0.02534 ; \lambda_{4}=-0.012883 ; \lambda_{5}=-0.53184$ and $\lambda_{6}=-1.5832$, demonstrating the presence of the chaos with the two Lyapunov exponent positive [4]. The total time for Lyapunov exponents computation was the $\Delta \tau=150,000$ with time-step of $\tau=0.5$.

Aiming to minimize vibrations and reduce the oscillatory motion caused in the system in the following section proposes the application of State-Dependent Riccati Equation (SDRE) to reduce this chaotic motion, see Figure 2b, to a 


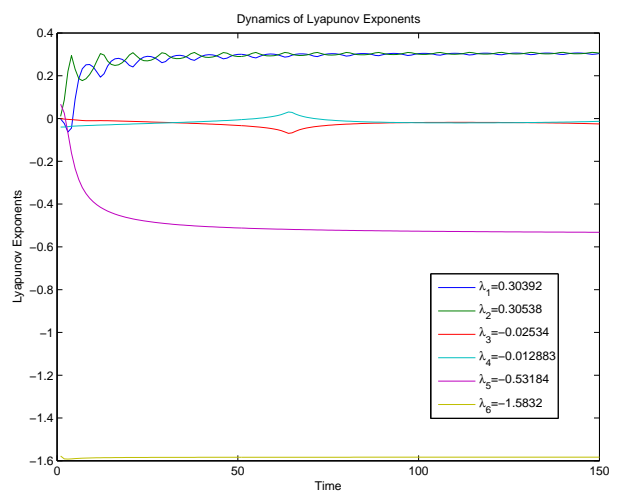

Figure 3: Dynamic of Lyapunov expoent

small stable orbit.

\section{Optimal Control Design}

The control objective is to stabilize the system with chaotic behavior in the small orbit periodic near the system origin. The Control Design via StateDependent Riccati Equation (SDRE) approach for obtaining a suboptimal solution of the control problem has the following procedure [9] and [10]:

1. Represent the model in state-space form. Use direct parametrization to bring the nonlinear dynamics $\dot{x}=f(x)+g(x)$ to the state-dependent coefficient (SDC) form, as follows:

$$
\begin{aligned}
& \dot{x}=A(x) x+B(x) u \\
& y=C(x) x
\end{aligned}
$$

where, $f(x)=A(x) x$ and $g(x)=B(x), A(x) \in \Re^{n \times n}$ is the dynamic matrix, $B(x) \in \Re^{n \times m}$ is the input matrix, $C(x) \in \Re^{s \times n}$ is the output matrix, $x \in \Re^{n}$ is the state vector $u \in \Re^{m}$ is the control law, $y \in \Re^{s}$ is the output vector.

2. Define the initial conditions $x(0)=x_{0}$, and choose the coefficients of positive definite weighting matrices $Q(x)$ and $R(x)$, which determine the relative importance of state $x(t)$ and control effort $u(t)$, respectively.

3. Solve the state-dependent Riccati equation given by:

$$
A^{T}(x) P(x)+P(x) A(x)-P(x) B(x) R^{-1}(x) B^{T}(x) P(x)+Q(x)=0
$$


4. Construct the nonlinear feedback control via:

$$
u=-R^{-1}(x) B^{T}(x) P(x) x
$$

The control law (5) is calculated so that the performance index given by, $J=\frac{1}{2} \int_{t_{0}}^{\infty}\left[x^{T} Q(x) x+u^{T} R(x) u\right] d t$ is minimized.

In the multivariable case, there always exists an infinite number of SDC parameterizations. Therefore, the choice of the matrix $A(x)$ isn't unique [9].

The pair $\{A(x), B(x)\}$ is a controllable parametrization of the nonlinear system in a region $\Omega$ if $\{A(x), B(x)\}$ is pointwise controllable in the linear sense for all $x \in \Omega$. Therefore, the choice of $A(x)$ must be such that the statedependent controllability matrix $\left[\begin{array}{llll}B(x) & A(x) B(x) & \ldots & A^{n-1}(x) B(x)\end{array}\right]$ has full rank [10].

The SDRE technique has been used to control various systems $[11,5]$. Details about the technique SDRE can be found in [9] and [10].

Applying the above procedure in the nonlinear system (2), the state dependent coefficients are given by:

$$
\begin{aligned}
& A_{(1,1)}=0 \\
& A_{(1,2)}=1 \\
& A_{(1,3)}=0 \\
& A_{(1,4)}=0 \\
& A_{(1,5)}=0 \\
& A_{(1,6)}=0 \\
& A_{(2,1)}=-\left(3\left(x_{1}-x_{3}\right)^{2}\right) / 400-\left(21 x_{1}^{2}\right) / 20 \\
& A_{(2,2)}=-1 / 250 \\
& A_{(2,3)}=\left(3 *\left(x_{1}-x_{3}\right)^{2}\right) / 400 \\
& A_{(2,4)}=1 / 500 \\
& A_{(2,5)}=\left(\cos \left(x_{5}\right) *\left(\left(3 x_{6}\right) / 2-29 / 10\right)\right) /\left(2 *\left(\sin \left(x_{5}\right)^{2} / 4-1\right)\right) \\
& -\left(x_{6}^{2} \sin \left(x_{5}\right)\right) / 2-\left(\cos \left(x_{5}\right) \sin \left(x_{5}\right)^{2}\right. \\
& \left.\left(\left(3 * x_{6}\right) / 2-29 / 10\right)\right) /\left(4\left(\sin \left(x_{5}\right)^{2} / 4-1\right)^{2}\right) \\
& A_{(2,6)}=x_{6} \cos \left(x_{5}\right)+\left(3 \sin \left(x_{5}\right)\right) /\left(4\left(\sin \left(x_{5}\right)^{2} / 4-1\right)\right) \\
& A_{(3,1)}=0
\end{aligned}
$$




$$
\begin{aligned}
& A_{(3,2)}=0 \\
& A_{(3,3)}=0 \\
& A_{(3,4)}=1 \\
& A_{(3,5)}=0 \\
& A_{(3,6)}=0 \\
& A_{(4,1)}=\left(3\left(x_{1}-x_{3}\right)^{2}\right) / 20 \\
& A_{(4,2)}=1 / 25 \\
& A_{(4,3)}=-\left(3\left(x 1-x_{3}\right)^{2}\right) / 20 \\
& A_{(4,4)}=-1 / 25 \\
& A_{(4,5)}=0 \\
& A_{(4,6)}=0 \\
& A_{(5,1)}=0 \\
& A_{(5,2)}=0 \\
& A_{(5,3)}=0 \\
& A_{(5,4)}=0 \\
& A_{(5,5)}=0 \\
& A_{(5,6)}=1 \\
& A_{(6,1)}=\left(\sin \left(x_{5}\right) *\left(k_{1}+k_{2}\right)\right) /\left(2\left(\sin \left(x_{5}\right)^{2} / 4-1\right)\right) \\
& A_{(6,2)}=0 \\
& A_{(6,3)}=-\left(k_{2} \sin \left(x_{5}\right)\right) /\left(2\left(\sin \left(x_{5}\right)^{2} / 4-1\right)\right) \\
& A_{(6,4)}=0 \\
& A_{(6,5)}=\left(\operatorname { c o s } ( x _ { 5 } ) \operatorname { s i n } ( x _ { 5 } ) \left(\sin \left(x_{5}^{2}\right) / 4-\left(\sin \left(x_{5}\right)\left(k_{1} x_{1}+k_{2}\left(x_{1}-x_{3}\right)\right)\right) / 2\right.\right. \\
& +\left(\sin \left(x_{5}\right)^{2} / 4-1\right)\left(\left(3 x_{6}\right) / 2-29 / 10\right)+ \\
& \left.\left.\left(x_{6}^{2} \cos \left(x_{5}\right) \sin \left(x_{5}\right)\right) / 4\right)\right) /\left(2\left(\sin \left(x_{5}\right)^{2} / 4-1\right)^{2}\right) \\
& -\left(\left(x_{5} \cos \left(x_{5}^{2}\right)\right) / 2-\left(\cos \left(x_{5}\right)\left(k_{1} x_{1}+k_{2}\left(x_{1}-x_{3}\right)\right)\right) / 2\right. \\
& +\left(x_{6}^{2} \cos \left(x_{5}\right)^{2}\right) / 4-\left(x_{6}^{2} \sin \left(x_{5}\right)^{2}\right) / 4 \\
& \left.+\left(\cos \left(x_{5}\right) \sin \left(x_{5}\right) *\left(\left(3 x_{6}\right) / 2-29 / 10\right)\right) / 2\right) /\left(\sin \left(x_{5}\right)^{2} / 4-1\right) \\
& A_{(6,6)}=-\left(\left(3 \sin \left(x_{5}\right)^{2}\right) / 8+\left(x_{6} \cos \left(x_{5}\right) \sin \left(x_{5}\right)\right) / 2-3 / 2\right) /\left(\sin \left(x_{5}\right)^{2} / 4-1\right)
\end{aligned}
$$




$$
B(x)=\left[\begin{array}{l}
1 \\
1 \\
1 \\
1 \\
1 \\
1
\end{array}\right]
$$

Assuming $C(x), Q(x)$ and $R(x)$ are constant matrices, we have:

$$
y=\left[\begin{array}{llllll}
1 & 0 & 0 & 0 & 0 & 0 \\
0 & 1 & 0 & 0 & 0 & 0 \\
0 & 0 & 1 & 0 & 0 & 0 \\
0 & 0 & 0 & 1 & 0 & 0 \\
0 & 0 & 0 & 0 & 1 & 0 \\
0 & 0 & 0 & 0 & 0 & 1
\end{array}\right]\left[\begin{array}{l}
x_{1} \\
x_{2} \\
x_{3} \\
x_{4} \\
x_{5} \\
x_{6}
\end{array}\right] \quad Q=\left[\begin{array}{llllll}
1 & 0 & 0 & 0 & 0 & 0 \\
0 & 1 & 0 & 0 & 0 & 0 \\
0 & 0 & 1 & 0 & 0 & 0 \\
0 & 0 & 0 & 1 & 0 & 0 \\
0 & 0 & 0 & 0 & 1 & 0 \\
0 & 0 & 0 & 0 & 0 & 1
\end{array}\right] \quad R=[1]
$$

The responses of the controlled system are shown in Figs. 4a and 4b .
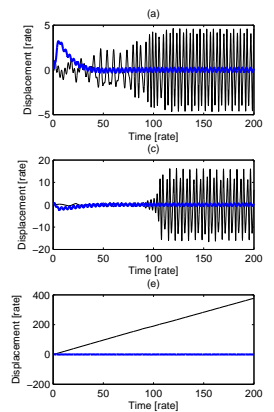

(a) Control Time History.
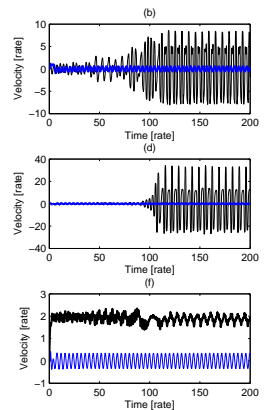

Figure 4: Control Dynamical System

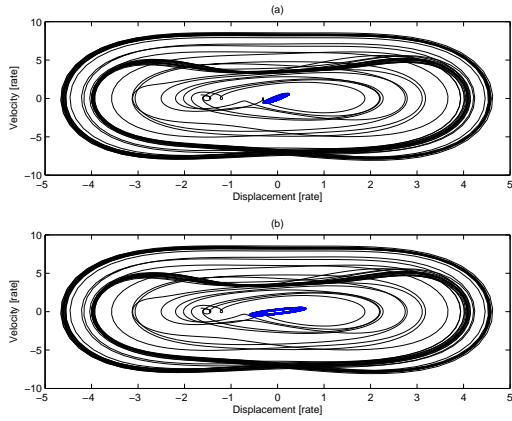

(b) Control Phase Portrait

\section{Conclusion}

In this paper the authors propose a model of a non-ideal vibration absorber. In section 2 the mathematical modeling is presented and computer simulations demonstrating the chaotic behavior of the system is shown in Figure 2 and 3. 
We proposed the use of an SDRE control strategy to reduce the chaotic movement of this system to a stable orbit. The Figures 4 illustrated the effectiveness of the control strategy taken to these problems.

\section{Acknowledgment}

The authors thanks all the support of the FAPESP (Proc $N^{o} .2014 / 16807-3$ ) and CNPq (Proc $\left.N^{o} .301769 / 2012-5\right)$.

\section{References}

[1] H. Frahm, Adevice for damping vibrations of bodies, US Patent 989958, 1911.

[2] V.O. Kononenko, Vibrating Systems with Limited Power Supply, Illife Books, London, 1969.

[3] R. Viquié, G. Kerschen, Nonlinear vibration absorber coupled to a nonlinear primary system: A tuning methodology. Journal of Sound and Vibration,326, (2009), 780-793, doi: 10.1016/j.jsv.2009.05.023.

[4] A. Wolf,, J.B. Swift, H.L. Swinney, J.A. Vastano, Determining Lyapunov Exponents from a Time Series. Physica D, 16, (1985), 285-317, doi: 10.1016/0167-2789(85)90011-9.

[5] F.R. Chavarette, Control design applied to a non-ideal structural system with behavior chaotic. International Journal of Pure and Applied Mathematics, 86-3, (2013), 487-500, doi: 10.12732/ijpam.v86i3.3 .

[6] B. S. C. Cunha, F.R. Chavarette. Vibration Control Applied in a SemiActive Suspension Using Magneto Rheological Damper and Optimal Linear Control Design. Applied Mechanics and Materials, 464, (2013), 229-234, doi: 10.4028/www.scientific.net/AMM.464.229.

[7] F.R. Chavarette, Optimal linear control to parametric uncertainties in a micro electro mechanical system. International Journal of Pure and Applied Mathematics, 83-4, (2013), 539-548, doi: 10.12732/ijpam.v83i4.2.

[8] F.R. Chavarette, On an Optimal Linear Control of a Chaotic Non-Ideal Duffing System. Applied Mechanics and Materials, 138-139, (2011), 50-55, doi: 10.4028/www.scientific.net/AMM.138-139.50. 
[9] J. R. Cloutier, C. N. D' Souza, C. P. Mracek, Nonlinear regulation and nonlinear $H_{\infty}$ control via the state-dependent Riccati equation technique, Proceedings of the International Conference on Nonlinear Problems in Aviation and Aerospace (1996), 117-142.

[10] C. P. Mracek, J. R. Cloutier, Control designs for the nonlinear benchmark problem via the state-dependent Riccati equation method, International Journal of Robust and Nonlinear Control, 8 (1998), 305-461, doi: 10.1002/(SICI)1099-1239(19980415/30)8:4/5<401::AIDRNC361>3.0.CO;2-U.

[11] A. Molter, M. Rafikov, Controle timo em agroecossistemas usando SDRE, Tendncias em Matemtica Aplicada e Computacional, 12, No. 3 (2011), 221-232, doi: 10.5540/tema.2011.012.03.0221. 